\title{
A new formula to get sharp global stability criteria for one-dimensional discrete-time models
}

\author{
Eduardo Liz • Sebastián Buedo-Fernández
}

December 20, 2018

\begin{abstract}
We present a new formula that makes it possible to get sharp global stability results for one-dimensional discrete-time models in an easy way. In particular, it allows to show that the local asymptotic stability of a positive equilibrium implies its global asymptotic stability for a new family of difference equations that finds many applications in population dynamics, economic models, and also in physiological processes governed by delay differential equations. The main ingredients to prove our results are the Schwarzian derivative and some dominance arguments.
\end{abstract}

Keywords Global stability, discrete-time model, Mackey-Glass equation, gamma-model, Schwarzian derivative.

Mathematics Subject Classification (2000) Primary 39A10, 39A30; Secondary $34 \mathrm{~K} 20$

\section{Introduction}

One of the central problems in dynamical systems modeling population or economic growth is the study of the existence and uniqueness of a positive equilibrium, and finding sufficient conditions under which all solutions converge to that equilibrium. For one-dimensional discrete-time models, existence and uniqueness are generally proved by elementary methods, and conditions for the local asymptotic stability are also easy to find in terms of the model parameters. In many cases, the local asymptotic stability of a unique positive equilibrium implies its global asymptotic stability (the folklore statement LAS implies GAS). However, although this property has already been suggested for some relevant population models in 1976

Eduardo Liz

Departamento de Matemática Aplicada II, Campus Marcosende, Universidad de Vigo, 36310 Vigo, Spain, E-mail: eliz@dma.uvigo.es

Sebastián Buedo-Fernández

Departamento de Estatística, Análise Matemática e Optimización, Universidade de Santiago de Compostela, Facultade de Matemáticas, Campus Vida, 15782 Santiago de Compostela, Spain E-mail: sebastian.buedo@usc.es 
$[12,20]$, the graphical analysis proposed there may work for particular examples but it does not provide a valid criterion to establish global stability conditions for a dynamical system depending on one or several parameters. After the pioneering results of Coppel [4], a very important step was given by Allwright [1] and Singer [26], with the use of the Schwarzian derivative: roughly speaking, for a $\mathcal{C}^{3}$ map $f$ decreasing or unimodal, with negative Schwarzian derivative and a unique fixed point, LAS implies GAS; for a precise statement, see [10, Theorem 1]. We recall that the Schwarzian derivative of a $\mathcal{C}^{3}$ map $F$ is defined by the expression

$$
(S F)(x)=\left(\frac{F^{\prime \prime \prime}(x)}{F^{\prime}(x)}\right)-\frac{3}{2}\left(\frac{F^{\prime \prime}(x)}{F^{\prime}(x)}\right)^{2},
$$

whenever $F^{\prime}(x) \neq 0$.

However, not all maps in population or economic models satisfy these conditions. Other useful tool is dominance or enveloping, which consists of comparing a map with another one that has the same equilibrium and it is known to be globally attracting. In this direction, see [5]. The approaches in [7] and [13] combine both tools (Schwarzian derivative and dominance).

Our main aim in this note consists of proving sharp global stability results for a family of difference equations which finds many applications in different fields such as population dynamics, economics, or physiological processes. We will refer to this family of difference equations as gamma-models, and they have the following general expression:

$$
x_{n+1}=x_{n}^{\gamma} F\left(x_{n}\right), \quad n=0,1,2, \ldots,
$$

where $\gamma \geq 0$, and $F:[0, \infty) \rightarrow(0, \infty)$ is a smooth function. Equation (1.1) defines a sequence $\left\{x_{n}\right\}_{n \geq 0}$ starting at any initial condition $x_{0}>0$.

The case $\gamma=1$ has attracted much attention in the framework of population dynamics, and in this case $F$ represents the per capita production function. In compensatory or overcompensatory models, $F$ is strictly decreasing (see, e.g., [11]). Sharp global stability criteria in this particular case can be found in many papers. Our results in this note follow the approach in [13], using also some tools from [7].

For $0 \leq \gamma<1$, and assuming that $F$ is decreasing, it is clear that (1.1) has a unique positive equilibrium. For $\gamma=1$, the existence of an equilibrium requires the extra assumptions $F(0)>1, F(\infty)<1$. If $\gamma>1$, then equation (1.1) can have more than one positive equilibria; in the latter case, it is a suitable model for populations with Allee effects (see, e.g., [14]). We will not consider this case, and will restrict ourselves to the case $0 \leq \gamma \leq 1$. For these values of $\gamma$, the use of equation (1.1) in population dynamics is intended to gain flexibility to fit population data. When $F(x)=\beta e^{-\delta x}, \beta, \delta>0,(1.1)$ provides the gamma-model included in the list of spawner-recruit models in the book of Quinn and Deriso [22] $]^{1}$. For example, Zheng and Kruse [28] found that this model fits well the stock-recruitment data for three Alaskan crab stocks; for more references, see [14].

Another interesting motivation comes from economics. Day [6] proposed to modify Solow's neoclassical growth model [27] introducing a production lag, which led him to express the model as a difference equation for the capital-labor ratio $x_{n}$ :

$$
x_{n+1}=\frac{s p\left(x_{n}\right) f\left(x_{n}\right)}{1-\lambda},
$$

\footnotetext{
1 For this reason we use the name gamma-models for (1.1).
} 
where $\lambda$ is the natural rate of population growth, $s$ is the (constant) savings ratio, $p(x)$ is the per capita production function, and $f(x)$ is a decreasing function which represents a "pollution effect" that reduces the productivity, and it is caused by increasing concentrations of capital.

Using the Cobb-Douglas production function $p(x)=B x^{\gamma}(B>0,0<\gamma<1)$, we arrive at equation (1.1) with $F(x)=B s(1-\lambda)^{-1} f(x)$.

It is worth mentioning that the global stability results for (1.1) provide sharp conditions for the global absolute stability of the positive equilibrium of the related family of delay differential equations

$$
x^{\prime}(t)=-\mu x(t)+x^{\gamma}(t-\tau) F(x(t-\tau)),
$$

with $\mu, \tau>0$. By absolute stability, we mean stability for all values of the delay (see [3] and references therein). We provide an example in Section 2.

The main finding in this paper is a new formula - stated as equation (2.1) which provides sharp global stability results in an easy way. This formula works in many cases when the criterion of the Schwarzian derivative does not work. In this way, it extends the classical Allwright-Singer result. To illustrate this fact, consider the following equivalent form of (2.1) suggested by Víctor Jiménez López:

$$
(S F)(x)<\frac{F(x)-x F^{\prime}(x)}{2(x F(x))^{2}}(x F(x))^{\prime}, \forall x>0 .
$$

According to our Theorem 1, condition (1.4) ensures the global asymptotic stability of the unique equilibrium $p$ of (1.1) if $F:[0, \infty) \rightarrow(0, \infty)$ is decreasing, $0 \leq \gamma \leq$ 1 , and $p$ is locally asymptotically stable. For $\gamma=0$, condition (1.4) is strictly weaker than the classical condition $(S F)(x)<0$ when $F$ is decreasing but $x F(x)$ is increasing.

For example, consider the map $F(x)=a /(1+x)^{m}$, with $a>0, m>0$, for which $(S F)(x)=\left(1-m^{2}\right) /\left(2(1+x)^{2}\right)$. If $m>1$, then $(S F)(x)<0$ for all $x>0$, and therefore the Allwright-Singer result applies. However, if $0<m \leq 1$, then $(S F)(x) \geq 0$ for all $x>0$, so this classical result cannot be applied. In this case, (1.4) holds because $x F(x)$ is increasing; thus our results ensure the global stability of the unique positive equilibrium $p$ (notice that $0>F^{\prime}(p)=-m p /(1+p)>-1$ if $0<m \leq 1)$.

Two important features of the central result in this note are the following: on the one hand, it is quite general, allowing to recover some known results in a unified and simpler way, and to prove some new relevant results for applications. On the other hand, it is easily verifiable, as we show in the applications. Therefore, we think it will be a useful tool for researchers interested in the global stability of difference equations and delay differential equations.

\section{Main result and examples}

For the main result in this section, we consider equation (1.1) with $0 \leq \gamma \leq 1$. Next we state the main assumptions for the map $F$.

(H) $F:[0, \infty) \rightarrow(0, \infty)$ is $\mathcal{C}^{3}$-differentiable and $F^{\prime}(x)<0$ for all $x>0$. For $\gamma=1$, we also assume that $F(0)>1, F(\infty)<1$. 
If $(\mathbf{H})$ holds, then there is a unique $p>0$ such that $F(p)=p^{1-\gamma}$, that is, $p$ is the unique positive equilibrium of (1.1).

We say that $p$ is a global attractor for (1.1) (or that $p$ is globally stable) if it is asymptotically stable and all solutions of (1.1) starting at an initial condition $x_{0}>0$ converge to $p$. We recall that an attracting fixed point of a continuous map of the real line is always stable [23, Corollary 2.1.3].

Theorem 1 Assume that $0 \leq \gamma \leq 1, F$ satisfies $(\mathbf{H})$, and the following condition holds:

$$
x^{2}\left(2(S F)(x)+\left(\frac{F^{\prime}(x)}{F(x)}\right)^{2}\right)<1, \forall x>0 .
$$

Then the local asymptotic stability of the unique positive equilibrium $p$ in equation (1.1) implies its global stability. This stability condition is

$$
-p^{\gamma} F^{\prime}(p) \leq 1+\gamma
$$

Remark. We notice that the stability condition (allowing for the non-hyperbolic cases) is $-1 \leq \gamma+p^{\gamma} F^{\prime}(p) \leq 1$, but $\gamma+p^{\gamma} F^{\prime}(p)$ is always less than 1 because $0 \leq \gamma \leq 1$ and $F^{\prime}(p)<0$. In this way, the stability condition is reduced to $(2.2)$.

We provide the proof of Theorem 1 in Section 4. Now we show its applicability with two classical examples from the literature about population dynamics. The second one gives absolute stability results for a generalization of the Mackey-Glass equation [18].

\section{Example 1.}

Consider the gamma-Ricker difference equation [14]:

$$
x_{n+1}=\beta x_{n}^{\gamma} e^{-\delta x_{n}},
$$

where $\beta, \delta>0,0 \leq \gamma \leq 1$. In this case, we easily get:

$$
F(x)=\beta e^{-\delta x} \quad, \quad(S F)(x)=-\frac{\delta^{2}}{2} \quad, \quad \frac{F^{\prime}(x)}{F(x)}=-\delta .
$$

Thus,

$$
2(S F)(x)+\left(\frac{F^{\prime}(x)}{F(x)}\right)^{2}=0, \forall x>0,
$$

and (2.1) trivially holds.

Next, condition (2.2) is equivalent to $p \delta \leq 1+\gamma$, which in turn is equivalent to the global stability condition (see [14]):

$$
\beta \leq e^{\gamma+1}\left(\frac{\gamma+1}{\delta}\right)^{1-\gamma}
$$

Thus, an application of Theorem 1 provides Theorem 1 (A) in [14]. For $\gamma=1$, we need the extra condition $\beta>1$, so we have the well-known global stability criterion $1<\beta \leq e^{2}$ for the Ricker map.

\section{Example 2.}

In this example we prove a global stability result for the difference equation 


$$
x_{n+1}=\frac{\beta x_{n}^{\gamma}}{1+\delta x_{n}^{m}},
$$

where $\beta, \delta, m>0,0 \leq \gamma \leq 1$. Equation (2.4) is a generalization of the population model proposed by Maynard Smith and Slatkin [21,25] (which is (2.4) with $\gamma=1$ ). We prove the following sharp global stability result:

Proposition 2 Assume that $\beta, \delta, m>0$ and $0 \leq \gamma \leq 1$. If $m \leq 1+\gamma$, then the unique positive equilibrium $p$ of (2.4) is globally asymptotically stable. If $m>1+\gamma$, then $p$ is globally asymptotically stable if and only if

$$
\beta \leq \frac{m}{m-1-\gamma}\left(\frac{1+\gamma}{\delta(m-1-\gamma)}\right)^{(1-\gamma) / m} .
$$

For $\gamma=1$, we have to assume that $\beta>1$ to ensure the existence of a positive equilibrium.

Proof Equation (2.4) is of the form of (1.1) with $F(x)=\beta /\left(1+\delta x^{m}\right)$. Since

$$
(S F)(x)=\frac{1-m^{2}}{2 x^{2}} \quad, \quad \frac{F^{\prime}(x)}{F(x)}=\frac{-\delta m x^{m-1}}{1+\delta x^{m}},
$$

we get

$$
x^{2}\left(2(S F)(x)+\left(\frac{F^{\prime}(x)}{F(x)}\right)^{2}\right)=1-m^{2}+m^{2}\left(\frac{\delta x^{m}}{1+\delta x^{m}}\right)^{2}<1, \forall x>0 .
$$

Thus, Theorem 1 ensures that $p$ is globally asymptotically stable if (2.2) holds. In this case, $(2.2)$ is equivalent to

$$
\frac{m \delta p^{m}}{1+\delta p^{m}} \leq 1+\gamma
$$

It is obvious that (2.6) is fulfilled if $m \leq 1+\gamma$. Next, we assume that $m>1+\gamma$. Solving (2.6) for $p$, we get the equivalent form

$$
p \leq\left(\frac{1+\gamma}{\delta(m-1-\gamma)}\right)^{1 / m} .
$$

Since $p \leq x$ if and only if $F(x) \leq x^{1-\gamma},(2.7)$ holds if and only if

$$
F\left(\left(\frac{1+\gamma}{\delta(m-1-\gamma)}\right)^{1 / m}\right) \leq\left(\frac{1+\gamma}{\delta(m-1-\gamma)}\right)^{(1-\gamma) / m}
$$

which is equivalent to $(2.5)$.

In the particular case $m=1$, equation (2.4) has been recently studied in [15], where it has been proved that the positive equilibrium is globally stable for all $\beta, \delta>0$ and $0<\gamma<1$. Thus, Proposition 2 provides a simpler proof of Theorem 3.1 in [15], and extends it to any $m \leq 1+\gamma$.

For $\gamma=1$, we get the sharp global stability condition $1<\beta \leq m /(m-2)$ for the Maynard Smith and Slatkin model. 
As an application to delay differential equations, we obtain a global stability result for the following generalization of the Mackey-Glass equation considered in [19]:

$$
x^{\prime}(t)=-\mu x(t)+\frac{\beta x^{\gamma}(t-\tau)}{1+x^{m}(t-\tau)},
$$

where $0 \leq \gamma \leq 1$. A well known result (see, e.g., [9]) establishes that if $p$ is a global attractor for the map

$$
H(x)=\frac{\beta x^{\gamma}}{\mu\left(1+x^{m}\right)},
$$

then $p$ is a global attractor for all positive solutions of (2.8) for all values of $\tau$.

Thus, we get the following corollary from Proposition 2 :

Corollary 3 The positive equilibrium of (2.8) is a global attractor for all values of the delay if either $m \leq 1+\gamma$, or $m>1+\gamma$ and

$$
\beta \leq \frac{\mu m}{m-1-\gamma}\left(\frac{1+\gamma}{m-1-\gamma}\right)^{(1-\gamma) / m} .
$$

For $\gamma=1$, we have to assume that $\beta>\mu$ to ensure the existence of a positive equilibrium.

In the classical paper [18], Mackey and Glass considered (2.8) with $\gamma=0$ and $\gamma=1$. In [8], Gopalsamy, Trofimchuk and Bantsur proved, among other results, the global stability criterion in Corollary 3 for these two particular cases. It is worth mentioning that they needed four auxiliary results ([8, Lemmas 1 to 4]) to get such a result, while we have stated and proved them in a unified way; furthermore, we have extended it for the whole parameter range $0 \leq \gamma \leq 1$.

3 The case $\gamma=0$

For the case $\gamma=0$, condition (2.1) gives a new formula to prove global stability when the sign of the Schwarzian derivative is not constant. Hence, it is worth formulating a more general result for this case. The proof is also given in Section 4.

Theorem 4 Assume that the $\mathcal{C}^{3}$ map $F:(0, \infty) \rightarrow(0, \infty)$ is either decreasing or unimodal (with a unique critical point $c$, which is a local extremum), and has a unique positive fixed point $p$, such that $F(x)>x$ if $x<p$ and $F(x)<x$ if $x>p$. If $F^{\prime}(p) \geq-1$ and (2.1) holds for all $x>0$ such that $F^{\prime}(x) \neq 0$, then $p$ is a global attractor for

$$
x_{n+1}=F\left(x_{n}\right), \quad n=0,1,2, \ldots
$$

We give an example that shows the applicability of Theorem 4:

\section{Example 3.}

In [17], the study of the absolute global stability of the positive equilibrium of a commodity market model governed by a delay differential equation was reduced to the global stability of the positive equilibrium of the following difference equation:

$$
x_{n+1}=\left(-b+\frac{a\left(d+x_{n}^{m}\right)}{c x_{n}^{m}}\right)^{1 / k}=: F\left(x_{n}\right),
$$


where $a, b, c, d, m>0, k \geq 1$, and $b c<a$. The map $F:(0, \infty) \rightarrow(0, \infty)$ is decreasing, and Lemma 2.5 in [17] ensures that $(S F)(x)<0$ holds for all $x>0$ if and only if $m>k$.

However, $F$ satisfies (2.1) also in the case $m \leq k$, so a direct application of Theorem 4 proves that the local asymptotic stability of the equilibrium implies its global stability. Indeed, direct computations lead to

$$
x^{2}\left(2(S F)(x)+\left(\frac{F^{\prime}(x)}{F(x)}\right)^{2}\right)-1=-\frac{(a-b c) m^{2} x^{m}\left(2 a d+(a-b c) x^{m}\right)}{\left(a d+(a-b c) x^{m}\right)^{2}}<0,
$$

since, by hypothesis, $a-b c>0$. In [17], the case $m \leq k$ was solved using Coppel's theorem [4].

The previous example corresponds to a monotone map $F$. A simple example involving a unimodal map is given by $F(x)=\beta \sqrt{x} e^{-x}$ (a particular case of (2.3)). It is easy to check that $(S F)$ does not have constant sign on $(0, \infty)$. However,

$$
x^{2}\left(2(S F)(x)+\left(\frac{F^{\prime}(x)}{F(x)}\right)^{2}\right)=\frac{1-8 x}{(1-2 x)^{2}}<1, \forall x>0, x \neq 1 / 2 .
$$

Hence, Theorem 4 guarantees that the condition $\beta^{2} \leq 3 e^{3} / 2$ for the local asymptotic stability of the positive equilibrium implies its global stability.

An important remark is that for some difference equations both Theorem 1 and Theorem 4 can be applied. In these cases, Theorem 1 is usually easier to use. An additional example showing that Theorem 1 works in some situations which do not fall into the scope of Theorem 4, let us consider the generalized gamma-Ricker difference equation (see, e.g., [13] for the case $\gamma=1$ ):

$$
x_{n+1}=x_{n}^{\gamma}\left(\alpha+(1-\alpha) e^{r\left(1-x_{n}\right)}\right)=: x_{n}^{\gamma} F\left(x_{n}\right),
$$

where $r>0,0 \leq \gamma \leq 1,0 \leq \alpha<1$. Since the map $f(x)=x^{\gamma} F(x)$ can have two critical points, Theorem 4 does not always apply. However, one can check that

$$
2(S F)(x)+\left(\frac{F^{\prime}(x)}{F(x)}\right)^{2}=-r^{2}+r^{2}\left(\frac{(1-\alpha) e^{r(1-x)}}{\alpha+(1-\alpha) e^{r(1-x)}}\right)^{2} \leq 0, \forall x>0,
$$

and therefore (2.1) holds.

\section{Proofs}

The main ingredient to prove Theorem 1 is the following generalization of Theorem 2.3 in [13], where the case $\gamma=1$ has been considered.

Theorem 5 Assume that $0<\gamma \leq 1$ and there exist constants $a>0, b \geq 0$ such that

$$
r(y)<g(y)<0, \forall y>0 \quad \text { and } \quad 0<g(y)<r(y), \forall y \in(-1 / b, 0),
$$

where $r(y)=-a y /(1+b y)$, and we mean $-1 / b=-\infty$ in case $b=0$.

If $\gamma-a \geq-1$, then the equilibrium $y=0$ is globally stable for equation

$$
y_{n+1}=\gamma y_{n}+g\left(y_{n}\right)
$$


Proof Assume first that $b>0$. By using the change of variables $t_{n}=b y_{n}$, we can suppose without restriction that $b=1$; thus, we assume that $r(y)=-a y /(1+y)$. Next, we consider the auxiliary equation

$$
y_{n+1}=\gamma y_{n}+r\left(y_{n}\right):=R\left(y_{n}\right),
$$

where the map $R(x)=\gamma x-a x /(1+x)$ is defined for all $x>-1$. It is easy to check that $R$ has a unique critical point $z=-1+\sqrt{a / \gamma}$, at which it attains a global minimum. To ensure that (4.3) is well defined, we have to prove that $R(z)>-1$. After some computations, we have

$$
R(z)=-(\sqrt{a}-\sqrt{\gamma})^{2} .
$$

Since, by hypothesis, $\gamma-a \geq-1$, we get

$$
1+\sqrt{\gamma}>\sqrt{1+\gamma} \geq \sqrt{a} \Longrightarrow \sqrt{a}-\sqrt{\gamma}<1 \Longrightarrow R(z)>-1,
$$

where we have used that $1+\sqrt{x}>\sqrt{1+x}$ for all $x \in(0,1]$.

Next, we have

$$
(S R)(x)=\frac{-6 a \gamma}{\left(a-\gamma(1+x)^{2}\right)^{2}}<0, \forall x \neq z .
$$

Since $R^{\prime}(0)=\gamma-a$, then $-1 \leq R^{\prime}(0)<1$ and therefore Corollary 2.10 in [7] ensures that 0 is a global attractor for equation (4.3).

Now, (4.1) implies that

$R(y)<\gamma y+g(y)<\gamma y \leq y, \forall y>0 \quad$ and $\quad y \leq \gamma y<\gamma y+g(y)<R(y), \forall y \in(-1,0)$.

The result in case $b>0$ follows from Theorem B in [7].

Next we assume that $b=0$. Then condition (4.1) implies that

$$
(\gamma-a) y<\gamma y+g(y)<\gamma y \leq y, \forall y>0,
$$

and

$$
y \leq \gamma y<\gamma y+g(y)<(\gamma-a) y, \forall y \in(-1,0) .
$$

Since $-1 \leq \gamma-a<1$, it is easy to check that the map $\gamma x+g(x)$ cannot have 2-periodic points different from 0 , and hence 0 is globally stable for (4.2) (see, e. g., the main theorem in [4]). We notice that, although this global stability criterion is usually stated for maps $\varphi:[A, B] \rightarrow[A, B]$, where $[A, B]$ is a compact interval, in this case, for any initial condition $x_{0} \in \mathbb{R}$ it is clear that all iterations of the map $\varphi(x)=\gamma x+g(x)$ starting at $x_{0}$ remain in the interval $\left[-x_{0}, x_{0}\right]$, so we can apply Coppel's result.

To prove Theorem 1, we use a change of variables which transforms (1.1) into (4.2), with $g(x)=-\ln \left(p^{\gamma-1} F\left(p e^{-x}\right)\right)$. Actually, formula (2.1) comes from this change of variables, as the following result shows.

Proposition 6 Assume that $0 \leq \gamma \leq 1$ and $F$ satisfies $(\mathbf{H})$. Let $p$ be the unique positive equilibrium of (1.1), and define

$$
g(x)=-\ln \left(p^{\gamma-1} F\left(p e^{-x}\right)\right) .
$$

Then, condition (2.1) is equivalent to $(S g)(x)<0$ for all $x \in \mathbb{R}$. 
Proof We use the formula for the Schwarzian derivative of the composition of two maps (see, e.g., [26]):

$$
(S(\phi \circ \psi))(x)=(S \phi)(\psi(x))\left(\psi^{\prime}(x)\right)^{2}+(S \psi)(x) .
$$

It is clear that $(S g)(x)<0$ if and only if $(S h)(x)<0$, where $h(x)=\ln \left(F\left(p e^{-x}\right)\right)$. Using (4.5), and taking into account that $\left(S f_{1}\right)(x)=-1 / 2$ for $f_{1}(x)=e^{-x}$, and $\left(S f_{2}\right)(x)=1 /\left(2 x^{2}\right)$ for $f_{2}(x)=\ln (x)$, we get

$$
(S h)(x)=\frac{-1}{2}+\left(p e^{-x}\right)^{2}\left[(S F)\left(p e^{-x}\right)+\frac{1}{2}\left(\frac{F^{\prime}\left(p e^{-x}\right)}{F\left(p e^{-x}\right)}\right)^{2}\right] .
$$

Hence, writing $p e^{-x}=y$, we have:

$$
(S h)(x)<0, \forall x \in \mathbb{R} \Longleftrightarrow \frac{-1}{2}+y^{2}\left[(S F)(y)+\frac{1}{2}\left(\frac{F^{\prime}(y)}{F(y)}\right)^{2}\right]<0, \forall y>0,
$$

and the last inequality is equivalent to (2.1).

Now we are in a position to prove Theorems 1 and 4 .

Proof (Proof of Theorem 1) The change of variables $y_{n}=-\ln \left(x_{n} / p\right)$ transforms (1.1) into (4.2), with $g(x)=-\ln \left(p^{\gamma-1} F\left(p e^{-x}\right)\right)$. By Proposition 6, condition (2.1) implies that $(S g)(y)<0$, for all $y \in \mathbb{R}$. Since $F$ is decreasing, it is clear that $g$ is decreasing. In order to apply Theorem 5 , we distinguish three cases:

- Case 1: If $g^{\prime \prime}(0)=0$, then $(S g)(0)=g^{\prime \prime \prime}(0) / g^{\prime}(0)<0$, and therefore $g^{\prime \prime \prime}(0)>$ 0 . The negativity of the Schwarzian derivative ensures that 0 is the unique inflexion point of $g$ (see, e. g., [24, Property 3, p. 141]); moreover, $g^{\prime}$ decreases on $(-\infty, 0)$ and increases on $(0, \infty)$. Then, it follows that $(4.1)$ holds with $a=-g^{\prime}(0)>0$ and $b=0$.

- Case 2: If $g^{\prime \prime}(0)>0$, then Lemma 2.1 in [16] implies that (4.1) holds with $a=-g^{\prime}(0)>0$ and $b=-g^{\prime \prime}(0) /\left(2 g^{\prime}(0)\right)>0$. Thus, the result when $g^{\prime \prime}(0) \geq 0$ follows from Theorem 5 , having in mind that $\gamma-a=\gamma+g^{\prime}(0)=\gamma+p^{\gamma} F^{\prime}(p)$.

- Case 3: If $g^{\prime \prime}(0)<0$, then the change of variables $z_{n}=-y_{n}$ transforms (4.2) into

$$
z_{n+1}=\gamma z_{n}+h\left(z_{n}\right),
$$

with $h(z)=-g(-z)$. Then, Lemma 2.1 in [16] can be applied to the map $h$ (see [13, Corollary 2.7] for more details). In this way, case 3 is reduced to case 2 , and the proof is complete.

Finally, the proof of the case $\gamma=0$ (and of Theorem 4) follows easily:

Proof (Proof of Theorem 4) The change of variables $y_{n}=-\ln \left(x_{n} / p\right)$ transforms (3.1) into

$$
y_{n+1}=g\left(y_{n}\right)
$$

with $g(x)=-\ln \left(p^{-1} F\left(p e^{-x}\right)\right)$. It is easy to check that $g$ is decreasing or unimodal. Since $(2.1)$ ensures that $(S g)(x)<0$ for all $x$ different from the critical point $c^{\prime}=\ln (p / c)$, Corollary 2.10 in [7] ensures that 0 is a global attractor for equation (4.7) if $-1 \leq g^{\prime}(0)<0$, which is equivalent to $-1 \leq F^{\prime}(p)<0$. If $F^{\prime}(p) \geq 0$, then the global stability of $p$ follows from the negative feedback condition $F(x)>x$ if $x<p$, and $F(x)<x$ if $x>p$. 


\section{Discussion}

There are two issues that we would like to emphasize. First, we have introduced a new family (1.1) of difference equations that provide great flexibility to fit population data and are also suitable as discrete-time models in economics; moreover, they can display a very rich dynamics (see, e.g., $[6,14,15]$ ). In particular, it seems that the issue of global stability for equation (2.4) has been studied for the first time in this paper; other properties have been stated in [19] in the framework of delay differential equations. Since the model of Maynard Smith and Slatkin has been already proved to be very flexible in population dynamics [2], we think that (2.4) has a great potential use in applications.

Second, we have been able to prove a global stability result valid for many different choices of the map $F$, including, for the case $\gamma=1$, many classical models. Our examples show that the conditions of Theorem 1 are easily verifiable. Thus, although Theorem 1 was already known in the case $\gamma=1$, the new formula (2.1) and its equivalent form (1.4) make the result more friendly. Even in the case $\gamma=0$, this formula provides a useful tool for proving the folklore statement LAS implies GAS in some cases where the Schwarzian derivative has not constant sign, and therefore Singer's result does not apply. To finish, we include the expression of (2.1) without invoking the Schwarzian derivative:

$$
x^{2}\left(2 \frac{F^{\prime \prime \prime}(x)}{F^{\prime}(x)}-3\left(\frac{F^{\prime \prime}(x)}{F^{\prime}(x)}\right)^{2}+\left(\frac{F^{\prime}(x)}{F(x)}\right)^{2}\right)<1,
$$

for all $x>0$ such that $F^{\prime}(x) \neq 0$.

\section{Acknowledgments}

The authors sincerely thank Víctor Jiménez López (Universidad de Murcia, Spain) and Ábel Garab (Alpen-Adria-Universität Klagenfurt, Austria) for useful discussions, encouraging comments and relevant remarks, and an anonymous reviewer for his/her helpful comments.

Eduardo Liz acknowledges the support of the research grant MTM2017-85054C2-1-P (AEI/FEDER, UE). The research of Sebastián Buedo-Fernández has been partially supported by Ministerio de Educación, Cultura y Deporte of Spain (grant number FPU16/04416), Consellería de Cultura, Educación e Ordenación Universitaria, Xunta de Galicia (grant numbers GRC2015/004 and R2016/022), and Agencia Estatal de Investigación of Spain (grant MTM2016-75140-P, cofunded by European Community fund FEDER).

\section{References}

1. D. J. Allwright, Hypergraphic functions and bifurcations in recurrence relations, SIAM J. Appl. Math. 34 (1978), 687-691.

2. T. S. Bellows, The descriptive properties of some models for density dependence, J. Anim. Ecol. 50 (1981), 139-156.

3. S. Buedo-Fernández, E. Liz, On the stability properties of a delay differential neoclassical model of economic growth, Electron. J. Qual. Theory Differ. Equ. 43 (2018), 1-14. 
4. W. A. Coppel, The solution of equations by iteration, Proc. Camb. Philos. Soc. 51 (1955), 41-43.

5. P. Cull, Population models: stability in one dimension, Bull. Math. Biol. 69 (2007), 9891017.

6. R. H. Day, Irregular growth cycles, Am. Econ. Rev. 72 (1982), 406-414.

7. H. A. El-Morshedy, V. Jiménez López, Global attractors for difference equations dominated by one-dimensional maps, J. Difference Equ. Appl. 14 (2008), 391-410.

8. K. Gopalsamy, S.I. Trofimchuk and N.R. Bantsur, A note on global attractivity in modems of hematopoiesis, Ukrain. Math. J. 50 (1998), 5-12.

9. A. F. Ivanov, A. N. Sharkovsky, Oscillations in singularly perturbed delay equations, Dynam. Report. Expositions Dynam. Systems (N.S.) 1 (1992), 164-224.

10. V. Jiménez López, E. Parreño, L.A.S. and negative Schwarzian derivative do not imply G.A.S. in Clark's equation, J. Dynam. Differential Equations 28 (2016), 339-374.

11. M. Kot, Elements of Mathematical Ecology, Cambridge University Press, New York, 2001.

12. S. A. Levin, R. M. May, A note on difference delay equations, Theor. Pop. Biol. 9 (1976), 178-187.

13. E. Liz, Local stability implies global stability in some one-dimensional discrete singlespecies models, Discrete Contin. Dyn. Syst., Ser. B 7 (2007), 191-199.

14. E. Liz, A global picture of the gamma-Ricker map: a flexible discrete-time model with factors of positive and negative density dependence, Bull. Math. Biol. 80 (2018), 417-434.

15. E. Liz, A new flexible discrete-time model for stable populations, Discrete Contin. Dyn. Syst. B 23 (2018), 2487-2498.

16. E. Liz, M. Pinto, G. Robledo, S. Trofimchuk, and V. Tkachenko, Wright type delay differential equations with negative Schwarzian, Discrete Contin. Dyn. Syst. 9 (2003), 309-321.

17. E. Liz, G. Röst, Global dynamics in a commodity market model, J. Math. Anal. Appl. 398 (2013), 707-714.

18. M.C. Mackey, L. Glass, Oscillation and chaos in physiological control system, Science 197 (1977), 287-289.

19. J. Mallet-Paret, R. D. Nussbaum, A differential-delay equation arising in optics and physiology, SIAM J. Math. Anal. 20 (1989), 249-292.

20. R. M. May, G. F. Oster, Bifurcations and dynamic complexity in simple ecological models, Am. Nat. 110 (1976), 573-599.

21. J. Maynard Smith, M. Slatkin, The stability of predator-prey systems, Ecology 54 (1973), 384-391.

22. T. J. Quinn, R. B. Deriso, Quantitative Fish Dynamics, Oxford University Press, New York, 1999.

23. H. Sedaghat, Nonlinear Difference Equations: Theory with Applications to Social Science Models, Mathematical Modelling: Theory and Applications, Vol. 15, Kluwer Academic Publishers, Dordrecht, 2003.

24. A. N. Sharkovsky, S. F. Kolyada, A. G. Sivak, V. V. Fedorenko, Dynamics of onedimensional maps, Mathematics and its Applications, 407, Kluwer Academic Publishers, Dordrecht, 1997.

25. J. G. Shepherd, A versatile new stock-recruitment relationship for fisheries, and the construction of sustainable yield resources, J. Conserv. Int. Explor. Mer. 40 (1982), 67-75.

26. D. Singer, Stable orbits and bifurcation of maps of the interval, SIAM J. Appl. Math. 35 (1978), 260-267.

27. R. M. Solow, A contribution to the theory of economic growth, Q. J. Econ. 70 (1956), 65-94.

28. J. Zheng, G. H. Kruse, Stock-recruitment relationships for three major Alaskan crab stocks, Fish. Res. 65 (2003), 103-121. 